JOURNAL CLUB

\title{
Alternatives to warfarin for the treatment of pulmonary emboli: the EINSTEIN-PE Study
}

The last few years have seen new oral anticoagulant treatments emerge with a major advantage over conventional vitamin $\mathrm{K}$ antagonism with warfarin: they have a predictable dose response that negates the need for laboratory monitoring. Dabigatran (Pradaxa), a direct thrombin inhibitor, and rivaroxaban are two such medications licenced for the prevention of stroke in atrial fibrillation. Rivaroxaban (Xiralto) is a direct inhibitor of Factor Xa. The 2011 ROCKET-AF study showed its efficacy in stroke prevention. ${ }^{1}$

The EINSTEIN-PE study was designed to compare efficacy and safety of fixed-dose oral rivaroxaban therapy against standard heparin plus warfarin in the treatment of symptomatic pulmonary emboli. ${ }^{2}$ This Bayer-sponsored study was randomised, open-label and included 4833 patients, half of whom were given $15 \mathrm{mg}$ rivaroxaban twice daily for 3 weeks, and $20 \mathrm{mg}$ once daily thereafter, and half of whom received long-term warfarin and enoxaparin at $1 \mathrm{mg} /$ $\mathrm{kg}$ until their international normalised ratio (INR) exceeded 2 for 2 days. The primary efficacy outcome of recurrent venous thromboembolism (VTE) occurred in 50 patients $(2.1 \%)$ taking rivaroxaban and 44 patients $(1.8 \%)$ with standard therapy. The secondary safety outcome of major bleeding (haemoglobin drop of $2 \mathrm{~g} / \mathrm{dl}$; a 2-unit transfusion; or bleeding intracranially, retroperitoneally or contributing to death) occurred in 26 rivaroxaban patients $(1.1 \%)$ and 52 standard-therapy patients $(2.2 \%)$. Contraindications to rivaroxaban included creatinine clearance of $<30 \mathrm{ml} / \mathrm{min}$, pregnancy and breastfeeding. The authors conclude that this evidence supports the use of rivaroxaban in the treatment of VTE, being as effective as and potentially safer than warfarin therapy.

Though dabigatran has been proven similarly effective by the RE-COVER trial, ${ }^{3}$ neither rivaroxaban nor dabigatran have yet been licenced for VTE treatment. If they are, choosing between them will be difficult due to the lack of head-to-head trials, though it is worth noting that while both are renally excreted and removable through dialysis, only rivaroxaban has a proven reversal agent, prothrombin complex concentrate, known to immediately and completely reverse its anticoagulant effect.

\section{Gareth Hynes}

Correspondence to Gareth Hynes, CT2, Royal Devon and Exeter Hospital, Exeter EX2 5DW, UK; gareth.hynes@gmail.com

Competing interests None.

Provenance and peer review Not commissioned; internally peer reviewed.

To cite Hynes G. Thorax 2013;68:503.

Published Online First 27 July 2012

Thorax 2013;68:503. doi:10.1136/thoraxjnl-2012-202321

\section{REFERENCES}

1 Patel MR, Mahaffey KW, Garg J. Rivaroxaban versus warfarin in Nonvalvular atrial Fibrillation. N Engl J Med 2011;365:883-91.

2 The EINSTEIN-PE investigators. Oral rivaroxaban for the treatment of symptomatic pulmonary embolism. N Engl J Med 2012;366:1287-97.

3 Schulman S, Kearon C, Kakkar AK. Dabigatran versus warfarin in the treatment of acute venous thromboembolism. N Engl J Med 2009:361:2342-52. 